\title{
Laboratory-based Prediction of Drug Biopharmaceutical Properties
}

\author{
Noha Mohamed Zaki Rayad* \\ Noha Mohamed Zaki Rayad, PhD, lecturer of Pharmaceutics, Faculty of Pharmacy, Ain Shams University, Abbassia, Cairo, Egypt
}

Bioavailability (BA), i.e. the amount of drug that is absorbed from the gut and delivered intact to the systemic circulation, is a main concern in the drug development process. Pharmaceutical scientists are striving to achieve as high $\mathrm{BA}$ as possible (one that approaches that after intravenous bolus). Biopharmaceutics is concerned with the physicochemical properties of a drug, the formulation in which the drug is placed as well as the physiology of the human being that would eventually lead to a high BA. The key biopharmaceutical properties are: solubility of drug in the gastrointestinal (GI) fluids and its permeability through the intestinal mucosa.

The goal of connecting in vitro and in vivo behaviour of a drug product is an endless goal for research, industry and regulatory societies. It is important to highlight how far the science has progressed in this regard but also how far it has to go to achieve this goal. Early in the drug discovery process, the favourable biopharmaceutical drug properties were acknowledged especially after these properties were represented by the renowned Biopharmaceutical Classification System (BCS). The guidelines for the BCS were introduced by the Food and Drug Administration (FDA) to simplify the production of generic drugs (FDA 2000). The drug discovery process was inspired by the $\mathrm{BCS}$ to adopt the structure-property relationship (SQR) rather than the traditional paradigm of structure-activity relationship (SAR). In this context, the lead structures in the drug discovery process were not only optimized with regard to their pharmacological properties but equally important, their biopharmaceutical properties.

This approach was primarily intended to reduce the high attrition rate (as high as $40 \%$ ) of new drug candidates which fail in the clinical trial phases because of their poor pharmacokinetic (i.e. ADMET) properties not because of their poor efficacy. Investigators have greatly attempted to develop models for both solubility and permeability to predict the drug absorption to avoid failure of the new drug candidate shortly. Modelling of both solubility and permeability phenomena is complex. In silico (computational) models as well as artificial membranes and cell-based models were devised for permeability screening. On the other hand, solubility descriptors for size, lipophilicity and the surface areas of non-polar atoms were claimed as being the most important for predictive model development. Models built from several descriptors are regarded as multivariate; hence, multivariate statistics (defined as those methods that examine multiple variables simultaneously) were used. These models can be obtained by multiple linear or nonlinear regressions of the variables. More advanced treatment is provided by partial least square projection (PLS) to latent structures, which is suitable when handling datasets with few observations and several variables.

Later in the drug development process, the BCS approach is implemented but with different objective. When formulators experience problematic compounds particularly regarding solubility and dissolution in the gastrointestinal (GI) fluids, the physicochemical properties of the drug, described by different prognostic models could be helpful to pick up the formulation strategy that would eventually result in the highest BA possible.

The generic industry is paying a similar attention to the BCS from a bioequivalence $(\mathrm{BE})$ viewpoint. Bioequivalent products denote that after single dose product administration to healthy volunteers and analysis of the drug in the blood, the empirical parameters like AUC, Tmax and Cmax are almost identical for the two products. Scientists argue that such in-vivo bioequivalence testing is time-, effortmoneyand blood- consuming, hence, great efforts were exerted to predict exactly and precisely how $\mathrm{BE}$ testing that works in vivo system could be replaced by a laboratory- based predictive system for oral bioavailability e.g. dissolution testing. The FDA suggests that Class I compounds, i.e. compounds for which the maximum dose given orally is dissolved in $250 \mathrm{ml}$ fluid within the $\mathrm{pH}$ interval of $\mathrm{pH} \mathrm{1-7.5}$ and of which $>90 \%$ is absorbed, do not need to be studied clinically after minor changes in the formulation (i.e. biowaived from in vivo BE. Hence, regulatory organizations like FDA and EMEA have approved in-vitro dissolution testing as a surrogate to in-vivo BE testing for certain BCS classes of drugs (esp. Class I drugs with high solubility and permeability) in which an in vitro-in vivo correlations (IVIVC) were established if the dissolution rate is slower than the gastric emptying rate. On the other hand, Class II drugs (with low solubility and high permeability) an IVIVC would expect only if the in vitro dissolution rate is similar to the in vivo dissolution rate and the drug has a low dose. To expand the IVIVC, scientists demand a better dissolution testing and modelling that would simulate the actual gut situation in order. At the forefront, the choice of a suitable dissolution medium i.e. one that can mimic the in vivo dissolution, is crucial. National pharmacopoeias define various test media to cover the physiological $\mathrm{pH}$ range i.e. from 1.2 to 6.8. Nevertheless, these media and this $\mathrm{pH}$ range are not very useful for many poorly soluble drugs. Others argue that these are not physiological media; hence could not provide a good predictability of the in vivo performance of a dosage form. Modern dissolution media were launched namely fasted state simulated intestinal fluid (FaSSIF) and fed state simulated intestinal fluid (FeSSIF) that contain surfactants present in GIT like bile salts and lecithin that improve the wetting and solubility of many lipophilic substances. Furthermore, the interaction of intestinal juices with lipids or food components can increase the formation of micelles for solubilization of the poorly

*Corresponding author: Noha Mohamed Zaki Rayad, PhD, lecturer of Pharmaceutics, Faculty of Pharmacy, Ain Shams University, Abbassia, Cairo, Egypt, Tel: (+202) 24102107; Fax: (+202) 24032059; E-mail: anm1998@lycos.com

Received November 28, 2011; Accepted December 05, 2011; Published December 07, 2011

Citation: Zaki Rayad NM (2011) Laboratory-based Prediction of Drug Biopharmaceutical Properties. J Bioequiv Availab 3: x-xi. doi:10.4172/jbb.10000e10

Copyright: ( 2011 Zaki Rayad NM. This is an open-access article distributed under the terms of the Creative Commons Attribution License, which permits unrestricted use, distribution, and reproduction in any medium, provided the original author and source are credited. 
Citation: Zaki Rayad NM (2011) Laboratory-based Prediction of Drug Biopharmaceutical Properties. J Bioequiv Availab 3: x-xi. doi:10.4172/ jbb.10000e10

soluble drugs or emulsions that are absorbed through the lymphatic vessels. In vitro, besides physiological bile components, a number of synthetic surfactants were also explored with varying degree of success in simulating the in vivo dissolution. The potential of solubilization enhancement of pharmaceutical surfactants can be used to develop dissolution media which are able to simulate the in vivo environment to establish an IVIVIC.
Taken altogether, great endeavour has been dedicated to attain the vision of a laboratory-based predictive system for oral drug absorption through better solubility and permeability testing and modeling. Importantly, conversion of laboratory data into pharmacokinetic profiles that enhance the insight of what is actually occurring in vivo, is indeed appreciated at all stages of drug discovery and development. 